\title{
An Efficient Hybrid Numerical Scheme for Nonlinear Multiterm Caputo Time and Riesz Space Fractional-Order Diffusion Equations with Delay
}

\author{
A. K. Omran $\mathbb{D}^{1,2}$ M. A. Zaky $\mathbb{D}^{1,{ }^{3,4}}$ A. S. Hendy $\mathbb{D}^{1,5}$ and V. G. Pimenov $\mathbb{D}^{1,6}$ \\ ${ }^{1}$ Department of Computational Mathematics and Computer Science, Institute of Natural Sciences and Mathematics Ural \\ Federal University, 19 Mira St., Yekaterinburg 620002, Russia \\ ${ }^{2}$ Department of Mathematics, Faculty of Science, Al-Azhar University, Assiut 71524, Egypt \\ ${ }^{3}$ Department of Mathematics, Nazarbayev University, Nur-Sultan, Kazakhstan \\ ${ }^{4}$ Department of Applied Mathematics, Physics Division, National Research Centre, Dokki, Cairo 12622, Egypt \\ ${ }^{5}$ Department of Mathematics, Faculty of Science, Benha University, Benha 13511, Egypt \\ ${ }^{6}$ Institute of Mathematics and Mechanics, Ural Branch of the Russian Academy of Sciences, 16 Kovalevskoy St., \\ Yekaterinburg 620000, Russia
}

Correspondence should be addressed to M. A. Zaky; ma.zaky@yahoo.com and A. S. Hendy; a.s.khendi@urfu.ru

Received 27 October 2021; Revised 17 November 2021; Accepted 19 November 2021; Published 6 December 2021

Academic Editor: Youssri Hassan Youssri

Copyright (C) 2021 A. K. Omran et al. This is an open access article distributed under the Creative Commons Attribution License, which permits unrestricted use, distribution, and reproduction in any medium, provided the original work is properly cited.

In this paper, we construct and analyze a linearized finite difference/Galerkin-Legendre spectral scheme for the nonlinear multiterm Caputo time fractional-order reaction-diffusion equation with time delay and Riesz space fractional derivatives. The temporal fractional orders in the considered model are taken as $\left(0<\beta_{0}<\beta_{1}<\beta_{2}<\cdots<\beta_{m}<1\right)$. The problem is first approximated by the $L 1$ difference method on the temporal direction, and then, the Galerkin-Legendre spectral method is applied on the spatial discretization. Armed by an appropriate form of discrete fractional Grönwall inequalities, the stability and convergence of the fully discrete scheme are investigated by discrete energy estimates. We show that the proposed method is stable and has a convergent order of $2-\beta_{m}$ in time and an exponential rate of convergence in space. We finally provide some numerical experiments to show the efficacy of the theoretical results.

\section{Introduction}

Fractional-order partial differential equations have evolved into powerful tools for describing a wide range of anomalous behavior and complex systems in natural science and engineering $[1-8]$. In addition, time delay occurs frequently in realistic world and it has been considered in numerous mathematical models, e.g., automatic control systems with feedback and population dynamics. Moreover, fractional differential equations with delay have been used widely in a variety of scientific and technical disciplines, including the study of natural phenomena, mathematical modelling, and the studies of porous media $[9,10]$. Recently, a two-term time-fractional differential equation that contains specific instances of the fractional diffusion-wave problem (see, for example, $[11,12])$ has been investigated in the literature.

In recent years, multiterm time-fractional differential equations have attracted the attention of many researchers. The ability of these equations to describe complex multirate physical phenomena is a motivating force behind their development (see, e.g., [13-15]). They were proposed to improve the modelling accuracy by accurately depicting the anomalous diffusion process [16], accurately modelling various types of viscoelastic damping [17], and accurately reproducing the unsteady flow of a fractional Maxwell fluid [18] and Oldroyd-B fluid [19]. Daftardar-Gejji and Bhalekar [20] considered the multiterm time-fractional diffusion wave equation with constant coefficients. Through the use of a 
domain decomposition technique, they were able to derive the linear and nonlinear diffusion-wave equations of the fractional order. Luchko [21] used an appropriate maximum principle and the Fourier technique to study the existence, uniqueness, and a priori estimates for the multiterm timefractional diffusion equation with variable coefficients. A new analytic technique for solving three types of multiterm time-space fractional advection diffusion equations with nonhomogeneous Dirichlet boundary conditions was proposed by Jiang et al. [22], based on Luchko's theorem and the equivalent relationship between the Laplacian operator and the Riesz fractional derivative. Ding and Jiang [23] used the technique of spectral representation of the fractional Laplacian operator in order to provide the analytical solutions for the multiterm time-space fractional advectiondiffusion equations with mixed boundary conditions. For the solution of initial-boundary value problems of multiterm time fractional diffusion equations, Li et al. [24] examined the well-posedness and long-time asymptotic behaviour of the equations. Zaky [25] constructed a Legendre spectral tau algorithm to deal with the multiterm time-fractional diffusion equations. Hendy [26] presented numerical treatment for solving a class of one-dimensional multiterm time-space fractional advection-diffusion equations with a temporal delay of the functional type. Hendy and De Staelen [27] developed a high-order numerical approximation approach for multiterm time convection diffusion wave equations with a nonlinear fixed time delay. To solve a coupled system of nonlinear multiterm time-space fractional diffusion equations over a nonuniform temporal mesh, Hendy and Zaky [28] developed an effective finite difference/spectral approach. Very recently, Zaky et al. [29] presented a discrete fractional Grönwall inequality that is consistent with the $L 2-1_{\sigma}$ to cope with the analysis of multiterm time-fractional partial differential equations. The key advantage of the proposed discrete Grönwall inequality over earlier efforts was that it can be utilised to provide optimal error estimates for multiterm fractional problems with nonlinear delay. Inspired by these inequalities, we can state and prove the convergence and stability estimates for our proposed fully discrete scheme. The discrete versions of Grönwall inequalities are of high concern in the numerical analysis of the numerical schemes for fractional differential equations [30,31].

Single-term fractional differential equations are often unable to describe some of the changing characteristics of the systems accurately. However, several multiterm fractional differential equations provide us with new tools to solve such problems. The multiterm time-fractional diffusion equation is useful not only for modelling the behaviour of viscoelastic fluids and rheological materials [32] but also for approximating distributed-order differential equations [33]. Hence, studies on the multiterm time-fractional differential equations have become important and useful for different applications. The multiterm time-fractional diffusion equation, whose weight function is taken into the linear combination of the Dirac $\delta$-functions, is an important special case of the time-fractional diffusion equation of distributed order. In this paper, we consider the numerical approximations to the following generalized nonlinear mul- titerm time-space fractional reaction-diffusion equations with delay:

$$
\begin{aligned}
\sum_{r=0}^{m} q_{r} \frac{\partial^{\beta_{r}} u}{\partial t^{\beta_{r}}}= & \kappa \frac{\partial^{\alpha} u}{\partial|x|^{\alpha}}+f(u(x, t), u(x, t-\tilde{s})) \\
& +g(x, t), \quad x \in \Omega, t \in I,
\end{aligned}
$$

endowed with initial-boundary conditions of the form

$$
\begin{cases}u(x, t)=\psi(x, t), & x \in \Omega, t \in[-\tilde{s}, 0], \\ u(a, t)=u(b, t)=0, & t \in I .\end{cases}
$$

Here, $\Omega=(a, b) \subset \mathbb{R}$ and $I=(0, T] \subset \mathbb{R}$ are space and time domains, respectively. We denote $\partial^{\beta_{r}} / \partial t^{\beta_{r}}$ as the Caputo fractional derivative with the fractional orders $(0<$ $\left.\beta_{0}<\beta_{1}<\beta_{2}<\cdots<\beta_{m}<1\right)$. The parameters $\kappa, s$ are positive constants. The parameters $q_{r}$ are absolutely positive. Also, $1<\alpha<2$ is the space fractional order. The left and right Riemann-Liouville fractional derivatives of order $\alpha(n-1<$ $\alpha<n)$ on the infinite domain [34] are defined as

$$
\begin{aligned}
{ }_{-\infty} D_{x}^{\alpha} u(x, t) & =\frac{1}{\Gamma(n-\alpha)} \frac{\partial^{n}}{\partial x^{n}} \int_{-\infty}^{x}(x-\tau)^{n-1-\alpha} u(\tau, t) d \tau, \\
{ }_{x} D_{\infty}^{\alpha} u(x, t) & =\frac{(-1)^{n}}{\Gamma(n-\alpha)} \frac{\partial^{n}}{\partial x^{n}} \int_{x}^{\infty}(\tau-x)^{n-1-\alpha} u(\tau, t) d \tau,
\end{aligned}
$$

where $\Gamma(x)$ is the gamma function. Thus, the space fractional derivative in the Riesz form on the space interval $\Omega$ can be defined as [35]

$$
\begin{aligned}
\frac{\partial^{\alpha} u}{\partial|x|^{\alpha}} & =-c_{\alpha}\left({ }_{a} D_{x}^{\alpha} u(x, t)+{ }_{x} D_{b}^{\alpha} u(x, t)\right), \\
c_{\alpha} & =\frac{1}{2 \cos (\pi \alpha / 2)}, 1<\alpha<2 .
\end{aligned}
$$

The Caputo derivative $\partial^{\beta} / \partial t^{\beta}$ is defined as

$$
\frac{\partial^{\beta}}{\partial t^{\beta}} u(x, t)=\frac{1}{\Gamma(1-\beta)} \int_{0}^{t}(t-r)^{-\beta} \frac{\partial}{\partial r} u(x, r) d r, 0<\beta<1 .
$$

The main aim of this work is to construct and analyze an efficient linearized numerical scheme for the nonlinear multiterm Riesz space and Caputo time fractional reactiondiffusion problem with fixed delay. A hybrid numerical scheme combines the Galerkin-Legendre spectral schemes, and a uniform L1-type interpolation technique is designed. The main challenges of the considered work are represented in how to numerically approximate the time Caputo fractional derivative, Riesz space fractional derivatives, and the time delay to produce an easy-to-implement and consistent numerical scheme. Overcoming all of these challenges to yield a hybrid linear numerical scheme is a first target. The 
other target is to analyze the convergence and stability. The theoretical analysis of the constructed fully discrete scheme is successfully estimated using appropriate discrete fractional Grönwall inequalities, and the scheme is proven to be unconditionally stable and convergent.

The outline of this paper is as follows. In the next section, we will go over some essential definitions and properties of fractional derivative spaces, fractional Sobolev spaces, and Jacobi polynomials. The steps needed to construct a fully discrete scheme on a uniform mesh are detailed in Section 3. Some technical lemmas from the literature are summarized in Section 4. Furthermore, the stability and the convergence analyses of the fully discrete scheme are studied in Section 5. Finally, numerical experiments are performed in Section 6 to illustrate the convergence analysis of the proposed approach.

\section{Preliminaries}

We here give some essential fractional derivative spaces [36] and their required features which will be helpful in the coming analysis. After that, the definition of Jacobi polynomials and their basic properties are recalled. We now fix some notations for the sake of clearness:

(i) $(\cdot, \cdot)_{0, \Omega}$ is the inner product on the space $L^{2}(\Omega)$ with the $L^{2}$-norm $\|\cdot\|_{0, \Omega}$

(ii) The maximum norm is defined as $\|\cdot\|_{\infty}$

(iii) $C_{0}^{\infty}(\Omega)$ is the space of smooth functions with compact support in $\Omega$

(iv) $H^{r}(\Omega)$ and $H_{0}^{r}(\Omega)$ are the usual Sobolev spaces with the norm $\|\cdot\|_{r}$ and seminorm $|\cdot|_{r}$

(v) $\mathscr{P}_{N}(\Omega)$ is the space of polynomials defined on the domain $\Omega$ with degree at most $N$

(vi) The approximation space $\mathscr{W}_{N}^{0}$ is defined as $\mathscr{W}_{N}^{0}=$ $\mathscr{P}_{N}(\Omega) \cap H_{0}^{1}(\Omega)$

(vii) $I_{N}$ is the Legendre-Gauss-Lobatto interpolation operator $I_{N}: C(\bar{\Omega}) \longrightarrow \mathscr{W}_{N}$ as

$$
u\left(x_{k}\right)=I_{N} u\left(x_{k}\right) \in \mathscr{P}_{N}, \quad k=0,1, \cdots, N
$$

Definition 1. Fractional derivative spaces and their related norms and seminorms are defined as follows [36]:

(i) Left fractional space: let $\eta>0$. Define the seminorm $|u|_{J_{L}^{\eta}(\Omega)}=\left\|{ }_{a} D_{x}^{\eta} u\right\|_{0, \Omega}$ and the norm $\|u\|_{J_{L}^{\eta}(\Omega)}=$ $\left(|u|_{J_{L}^{\eta}}^{2}(\Omega)+\|u\|_{0, \Omega}^{2}\right)^{1 / 2}$, and let $J_{L}^{\eta}$ (or $J_{L, 0}^{\eta}$ ) denote the closure of $C^{\infty}(\Omega)$ (or $C_{0}^{\infty}(\Omega)$ ) with respect to $\|\cdot\|_{J_{L}^{\eta}(\Omega)}$

(ii) Right fractional space: let $\eta>0$. Define the seminorm $|u|_{J_{R}^{\eta}(\Omega)}=\left\|{ }_{x} D_{b}^{\eta} u\right\|_{0, \Omega}$ and the norm $\|u\|_{J_{R}^{\eta}(\Omega)}$
$=\left(|u|_{J_{R}^{\eta}(\Omega)}^{2}+\|u\|_{0, \Omega}^{2}\right)^{1 / 2}$, and let $J_{R}^{\eta}$ (or $J_{R, 0}^{\eta}$ ) denote the closure of $C^{\infty}(\Omega)$ (or $C_{0}^{\infty}(\Omega)$ ) with respect to $\|\cdot\|_{J_{R}^{\eta}(\Omega)}$

(iii) Symmetric fractional space: let $\eta \neq n-(1 / 2), n \in \mathbb{N}$. Define the seminorm $|u|_{J_{s}^{\eta}(\Omega)}=\left|\left({ }_{a} D_{x}^{\eta} u,{ }_{x} D_{b}^{\eta} u\right)_{0, \Omega}\right|^{1 / 2}$ and the norm $\|u\|_{J_{s}^{\eta}(\Omega)}=\left(|u|_{J_{s}^{\eta}}^{2}(\Omega)+\|u\|_{0, \Omega}^{2}\right)^{1 / 2}$, and let $J_{s}^{\eta}$ (or $J_{s, 0}^{\eta}$ ) denote the closure of $C^{\infty}(\Omega)$ (or $C_{0}^{\infty}(\Omega)$ ) with respect to $\|\cdot\|_{J_{s}^{\eta}(\Omega)}$

(iv) Fractional Sobolev space: let $\eta>0$. Define the fractional Sobolev space $H^{\eta}(\Omega)$ as $H^{\eta}(\Omega)=\left\{u \in L^{2}(\Omega)\right.$ $\left.\left.|| \omega\right|^{\eta} \mathscr{F}(\widehat{u}) \in L^{2}(\mathbb{R})\right\}$, endowed with the seminorm $|u|_{H^{\eta}(\Omega)}=\left\||\omega|^{\eta} \mathscr{F}(\widehat{u})\right\|_{0, \mathbb{R}}$ and the norm $\|u\|_{H^{\eta}(\Omega)}=$ $\left(|u|_{H^{\eta}(\Omega)}^{2}+\|u\|_{0, \Omega}^{2}\right)^{1 / 2}$, where $\mathscr{F}(\widehat{u})$ is the Fourier transformation of $\widehat{u}$ and $\widehat{u}$ is the extension of zero of $u$ outside $\Omega$. Denote by $H^{\eta}(\Omega)$ (or $H_{0}^{\eta}(\Omega)$ ) the closure of $C^{\infty}(\Omega)$ (or $C_{0}^{\infty}(\Omega)$ ) with respect to $\|\cdot\|_{H^{\eta}(\Omega)}$.

Lemma 2 (see [36]). The spaces $J_{L}^{\eta}, J_{R}^{\eta}, J_{s}^{\eta}$, and $H^{\eta}$ are equivalent, with equivalent seminorms and norms if $\eta \neq n-(1 / 2)$ ,$n \in \mathbb{N}$.

Lemma 3 (adjoint property). Let $1<\eta<2$, then for any $u \in$ $H_{0}^{\eta}(\Omega)$ and $v \in H_{0}^{\eta / 2}(\Omega)$, we get

$$
\begin{aligned}
\left({ }_{a} D_{x}^{\eta} u, v\right)_{0, \Omega} & =\left({ }_{a} D_{x}^{\eta / 2} u,{ }_{x} D_{b}^{\eta / 2} v\right)_{0, \Omega},\left({ }_{x} D_{x}^{\eta} u, v\right)_{0, \Omega} \\
& =\left({ }_{x} D_{x}^{\eta / 2} u,{ }_{a} D_{b}^{\eta / 2} v\right)_{0, \Omega} .
\end{aligned}
$$

Spectral methods are characterized by the expansion of the solution in terms of global and, usually, orthogonal polynomials [37-40]. Now, we present the Jacobi polynomials and some of their basic properties. The vital role in the field of spectral methods arose from the nature of Jacobi weights which are related to the singular kernels of time Caputo fractional derivatives of order $0<\beta<1$. Denote $J_{i}^{\mu, v}(x), \mu, v>-1$ as the $i$-th order Jacobi polynomial of index defined on $[-1,1]$. As all classic orthogonal polynomials, $\left\{J_{i}^{\mu, v}(x)\right\}_{i=0}^{N}$ satisfies the following three-termrecurrence relation:

$$
\left\{\begin{array}{l}
J_{0}^{\mu, v}(x)=1, \\
J_{1}^{\mu, v}(x)=\frac{1}{2}(2+\mu+v) x+\frac{1}{2}(\mu-v), \\
J_{i+1}^{\mu, v}(x)=\left(A_{i}^{\mu, v} x-B_{i}^{\mu, v}\right) J_{i}^{\mu, v}(x)-C_{i}^{\mu, v} J_{i-1}^{\mu, v}(x), \quad \text { if } 1 \leq i \leq N .
\end{array}\right.
$$


The recursion coefficients are given by

$$
\left\{\begin{array}{l}
A_{i}^{\mu, v}=\frac{(2 i+\mu+v+1)(2 i+\mu+v+2)}{2(i+1)(i+\mu+v+1)}, \\
B_{i}^{\mu, v}=\frac{(2 i+\mu+v+1)\left(v^{2}-\mu^{2}\right)}{2(i+1)(i+\mu+v+1)(2 i+\mu+v)}, \\
C_{i}^{\mu, v}=\frac{(2 i+\mu+v+2)(i+\mu)(i+v)}{(i+1)(i+\mu+v+1)(2 i+\mu+v)} .
\end{array}\right.
$$

Let $\omega^{\mu, \nu}(x)=(1-x)^{\mu}(1+x)^{\nu}$. Then, one has

$$
\int_{-1}^{1} J_{i}^{\mu, v}(x) J_{j}^{\mu, v}(x) \omega^{\mu, v}(t) d x=\gamma_{i}^{\mu, v} \delta_{i, j}, \quad \forall i=0,1, \cdots, N,
$$

where $\delta_{i, j}$ is the Kronecker delta function and

$$
\gamma_{i}^{\mu, v}=\frac{2^{(\mu+v+1)} \Gamma(i+\mu+1) \Gamma(i+v+1)}{(2 i+\mu+v+1) i ! \Gamma(i+\mu+v+1)}, \quad \forall i=0,1, \cdots, N
$$

In particular, the Legendre polynomial is defined as $L_{i}(x)=J_{i}^{0,0}(x)$.

\section{The Numerical Scheme}

Here, we provide a fully discrete scheme for the problems (1) and (2) based on the L1-type approximation for the Caputo time-fractional derivative and the Legendre-Galerkin spectral method in space. To discretize the time-fractional derivatives, we divide the interval $[0, T]$ uniformly with a time step size $\tau$ defined by $\tau=\tilde{s} / N_{\tilde{s}}$ such that $N_{\tilde{s}}$ is a positive integer. The uniform partitions given by $t_{n}=n \tau, \forall-N_{\tilde{s}} \leq n \leq M$, where $M=\lceil T / \tau\rceil$. The $L 1$ interpolation scheme for the time-fractional derivative of order $0<\beta_{r}<1, r=0,1,2, \cdots$, $m$, in the Caputo sense at the time $t_{n}$ is defined as

$$
\begin{aligned}
\left.\sum_{r=0}^{m} q_{r} \frac{\partial^{\beta_{r}} u}{\partial t^{\beta_{r}}}\right|_{t=t_{n}}= & \sum_{r=0}^{m} q_{r} \int_{0}^{t_{n}} u^{\prime}(x, \eta) \omega_{1-\beta_{r}}\left(t_{n}-\eta\right) d \eta \\
= & \sum_{r=0}^{m} \frac{q_{r}}{\Gamma\left(1-\beta_{r}\right)} \sum_{i=1}^{n} \frac{u\left(x, t_{i}\right)-u\left(x, t_{i-1}\right)}{\tau} \\
& \cdot \int_{t_{i-1}}^{t_{i}}\left(t_{n}-\eta\right)^{-\beta_{r}} d \eta+r_{\tau}^{n} \\
= & \sum_{r=0}^{m} \frac{q_{r}}{\Gamma\left(2-\beta_{r}\right) \tau^{\beta_{r}}} \sum_{i=1}^{n} a_{i}^{\beta_{r}} u\left(x, t_{i}\right) \\
& -u\left(x, t_{i-1}\right)+r_{\tau}^{n},
\end{aligned}
$$

where $\omega_{\beta_{r}}(t)=t^{\beta_{r}-1} / \Gamma\left(\beta_{r}\right), t>0$, and $a_{j}^{\beta_{r}}=(j+1)^{1-\beta_{r}}-j^{1-\beta_{r}}$, for each $j \geq 0$. If $u \in C^{2}\left([0, T] ; L^{2}(\Omega)\right)$, then there exists a constant $C>0$ such that the truncation error $r_{\tau}^{n}$ satisfies $\left\|r_{\tau}^{n}\right\| \leq C \tau^{2-\beta_{m}}$, for each $n=0,1, \cdots, M$ (see [41]).
Definition 4. Let $\left\{u^{n}\right\}_{n=0}^{M}$ be a sequence of real functions defined on $\Omega$. We define the multiterm discrete timefractional difference operator $\sum_{r=0}^{m} q_{r} D_{\tau}^{\beta_{r}}$ by

$$
\begin{aligned}
\sum_{r=0}^{m} q_{r} D_{\tau}^{\beta_{r}} u^{n} & =\sum_{r=0}^{m} \frac{q_{r}}{\Gamma\left(2-\beta_{r}\right) \tau^{\beta_{r}}} \sum_{i=1}^{n} a_{n-i}^{\beta_{r}} \delta_{t} u^{i} \\
& =\sum_{r=0}^{m} \frac{q_{r}}{\Gamma\left(2-\beta_{r}\right) \tau^{\beta_{r}}} \sum_{i=0}^{n} b_{n-i}^{\beta_{r}} u^{i}, \quad \forall n=1, \cdots, M .
\end{aligned}
$$

In this expression, $\delta_{t} u^{i}=u^{i}-u^{i-1}$, and the constants are defined by $b_{0}^{\beta_{r}}=a_{0}^{\beta_{r}}, b_{n}^{\beta_{r}}=-a_{n-1}^{\beta_{r}}$, and $b_{n-i}^{\beta_{r}}=a_{n-i}^{\beta_{r}}-a_{n-i-1}^{\beta_{r}}$, for each $i=1, \cdots, n-1$.

In order to provide a semidiscretized form of (1) at each time $t_{n}$, we approximate the time-fractional term through (13). Taylor approximations are used to approximate the nonlinear source function in a linear style. As a consequence, we obtain the discrete-time system:

$$
\begin{aligned}
\sum_{r=0}^{m} q_{r} D_{\tau}^{\beta_{r}} u^{n}= & \frac{\partial^{\alpha} u^{n}}{\partial|x|^{\alpha}}+f\left(2 u^{n-1}-u^{n-2}, u^{n-N_{\tilde{\mathrm{s}}}}\right) \\
& +g^{n}(x), \quad 1 \leq n \leq M, \forall x \in \Omega,
\end{aligned}
$$

$$
u^{n}(x)=\psi(x), \quad-N_{\tilde{s}} \leq n \leq 0, x \in \Omega
$$

We define the following function space to give appropriate base functions such that the boundary conditions are satisfied exactly as clarified in spectral methods for spacefractional differential equations [42, 43].

$$
\mathscr{W}_{N}^{0}=\operatorname{span}\left\{\varphi_{n}(x): n=0,1, \cdots, N-2\right\},
$$

where for each $\hat{x} \in[-1,1]$, the function $\varphi_{n}$ is given by

$$
\varphi_{n}(x)=L_{n}(\widehat{x})-L_{n+2}(\widehat{x})=\frac{2 n+3}{2(n+1)}\left(1-\widehat{x}^{2}\right) J_{n}^{1,1}(\widehat{x}),
$$

and $x=1 / 2((b-a) \hat{x}+a+b) \in[a, b]$.

We introduce the parameter $\sigma_{r}=q_{r} / \Gamma\left(2-\beta_{r}\right) \tau^{\beta_{r}}$. Then, the scheme (14) can be rewritten in the following equivalent form:

$$
\begin{aligned}
\sum_{r=0}^{m} \sigma_{r} a_{0}^{\beta_{r}} u^{n}-\kappa \frac{\partial^{\alpha} u^{n}}{\partial|x|^{\alpha}}= & \sum_{r=0}^{m} \sigma_{r} a_{n-1}^{\beta_{r}} u^{0}-\sum_{r=0}^{m} \sigma_{r} \sum_{i=1}^{n-1} b_{n-i}^{\beta_{r}} u^{i} \\
& +f\left(2 u^{n-1}-u^{n-2}, u^{n-N_{\tilde{s}}}\right) \\
& +g^{n}(x), \quad \forall n=1, \cdots, M
\end{aligned}
$$


The fully discrete $L 1$-Galerkin spectral scheme consists of the set of approximations $u_{N}^{n} \in \mathscr{W}_{N}^{0}$, satisfying the system:

$$
\left\{\begin{array}{l}
\sum_{r=0}^{m} \sigma_{r} a_{0}^{\beta_{r}}\left(u_{N}^{n}, v\right)-\kappa\left(\frac{\partial^{\alpha}}{\partial|x|^{\alpha}} u_{N}^{n}, v\right)=\sum_{r=0}^{m} \sigma_{r} a_{n-1}^{\beta_{r}}\left(u_{N}^{0}, v\right)-\sum_{r=0}^{m} \sigma_{r} \sum_{i=1}^{n-1} b_{n-i}^{\beta_{r}}\left(u_{N}^{i}, v\right)+\left(I_{N} f\left(2 u_{N}^{n-1}-u_{N}^{n-2}, u_{N}^{n-N_{\tilde{s}}}\right), v\right)+\left(I_{N} g^{n}(x), v\right), \quad \forall v \in \mathscr{W}_{N}^{0}, \forall n=1, \cdots, M, \\
u_{N}^{n}=\pi_{N}^{1,0} \psi\left(t_{n}, x\right), \quad-N_{\tilde{s}} \leq n \leq 0,
\end{array}\right.
$$

where $\pi_{N}^{1,0}$ is an appropriate projection operator. We expand the approximate solution as

$$
u_{N}^{n}=\sum_{i=0}^{N-2} \widehat{u}_{i}^{n} \varphi_{i}(x)
$$

Substituting this expression into (19) and letting $v=\varphi_{k}$, for each $0 \leq k \leq N-2$, we obtain the following matrix repre- sentation of the uniform $L_{1}$-Galerkin spectral scheme:

$$
\left(\sum_{r=0}^{m} \sigma_{r} a_{0}^{\beta_{r}} \bar{M}-\kappa c_{\alpha}\left(S+S^{T}\right)\right) U^{n}=K^{n-1}+R^{n-1}+F^{n} .
$$

The notations in this expression are given by the system of identities:

$$
\begin{cases}s_{i j}=\int_{\Omega}{ }_{a} D_{x}^{\alpha / 2} \varphi_{i}(x)_{x} D_{b}^{\alpha / 2} \varphi_{j}(x) d x, & S=\left(s_{i j}\right)_{i, j=0}^{N-2}, \\ m_{i j}=\int_{\Omega} \varphi_{i}(x) \varphi_{j}(x) d x, & \bar{M}=\left(m_{i j}\right)_{i, j=0}^{N-2}, \\ h_{i}^{n-1}=\int_{\Omega} \varphi_{i}(x) I_{N} f\left(2 u_{N}^{n-1}-u_{N}^{n-2}, u_{N}^{n-N_{s}}\right) d x, & R^{n-1}=\left(h_{0}^{n-1}, h_{1}^{n-1}, \cdots, h_{N-2}^{n-1}\right)^{\top}, \\ g_{i}^{n}=\int_{\Omega} \varphi_{i}(x) I_{N} g^{n} d x, & F^{n}=\left(g_{0}^{n}, g_{1}^{n}, \cdots, g_{N-2}^{n}\right)^{\top}, \\ U^{n}=\left(\widehat{u}_{0}^{n}, \widehat{u}_{1}^{n}, \cdots, \widehat{u}_{N-2}^{n}\right)^{\top}, & K^{n-1}=-\sum_{r=0}^{m} \sigma_{r} \sum_{j=0}^{n-1} b_{n-j}^{\beta_{r}} \bar{M} U^{j} .\end{cases}
$$

Lemma 5 (see $[42,43])$. The components of the stiffness matrix $S$ are $s_{i j}=a_{i}^{j}-a_{i}^{j+2}-a_{i+2}^{j}+a_{i+2}^{j+2}$, for each $i, j=0,1, \cdots$ , $N-2$. Here,

$$
\begin{aligned}
a_{i}^{j}= & \int_{\Omega}{ }_{a} D_{x}^{\alpha / 2} L_{i}(\widehat{x})_{x} D_{b}^{\alpha / 2} L_{j}(\hat{x}) d x \\
= & \left(\frac{b-a}{2}\right)^{1-\alpha} \frac{\Gamma(i+1) \Gamma(j+1)}{\Gamma(i-(\alpha / 2)+1) \Gamma(j-(\alpha / 2)+1)} \\
& \cdots \sum_{r=0}^{N} \omega_{r}^{-\alpha / 2,-\alpha / 2} J_{i}^{\alpha / 2,-\alpha / 2}\left(x_{r}^{-\alpha / 2,-\alpha / 2}\right) J_{j}^{-\alpha / 2, \alpha / 2}\left(x_{r}^{-\alpha / 2,-\alpha / 2}\right),
\end{aligned}
$$

and $\left\{x_{r}^{-\alpha / 2,-\alpha / 2}, \omega_{r}^{-\alpha / 2,-\alpha / 2}\right\}_{i=0}^{N}$ is the set of Jacobi-Gauss points and weights with respect to the weight function $\omega^{-\alpha / 2,-\alpha / 2}$. The mass matrix $\bar{M}$ is symmetric, with nonzero components:

$$
m_{i j}=m_{j i}= \begin{cases}\frac{b-a}{2 j+1}+\frac{b-a}{2 j+5}, & \forall i=j, \\ -\frac{b-a}{2 j+5}, & \forall i=j+2 .\end{cases}
$$

\section{Technical Lemmas}

Several lemmas that will be invoked through our analysis appeared in that section. In the sequel, $C$ and $C_{u}$ will denote generic positive constants independent of $\tau, N$, and $n$ and may be different under different circumstances. We also fix the following notation $\mathbb{Z}_{[a, b]}=\mathbb{Z} \cap[a, b]$, such that $\mathbb{Z}$ is the set of all integers. 
Throughout the coming context, we will use the notation

$$
A(u, w)=\kappa c_{\alpha}\left[\left({ }_{a} D_{x}^{\alpha / 2} u,{ }_{x} D_{b}^{\alpha / 2} w\right)+\left({ }_{x} D_{b}^{\alpha / 2} u,_{a} D_{x}^{\alpha / 2} w\right)\right]
$$

The orthogonal projection operator $\pi_{N}^{\alpha / 2,0}: H_{0}^{\alpha / 2}(\Omega)$ $\longrightarrow \mathscr{W}_{N}^{0}$ will be such that

$$
A\left(u-\pi_{N}^{\alpha / 2,0} u, w\right)=0, \quad \forall u \in H_{0}^{\alpha / 2}(\Omega), w \in \mathscr{W}_{N}^{0} .
$$

For convenience of theoretical analysis, we give the following seminorm and norm:

$$
\begin{aligned}
|u|_{\alpha / 2} & :=A(u, u)^{1 / 2}, \\
\|u\|_{\alpha / 2} & :=\left(\|u\|^{2}+|u|_{\alpha / 2}^{2}\right)^{1 / 2},
\end{aligned}
$$

which are equivalent to the seminorms and norms of $J_{L}^{\alpha / 2}(\Omega), J_{R}^{\alpha / 2}(\Omega), J_{S}^{\alpha / 2}(\Omega)$, and $H^{\alpha / 2}(\Omega)$. We recall the following three lemmas from [43].

Lemma 6. Let $\alpha$ and $s$ be arbitrary real numbers satisfying $0<\alpha<1, \alpha<s, \alpha \neq 1 / 2$. Then, there exists a positive constant $C$ independent of $N$ such that, for any function $u \in H_{0}^{\alpha / 2}(\Omega)$ $\cap H^{s}(\Omega)$, the following estimate holds:

$$
\left|u-\pi_{N}^{\alpha / 2,0} u\right|_{\alpha / 2} \leq C N^{\alpha / 2-s}\|u\|_{s} .
$$

Lemma 7. Suppose that $\Omega=(a, b), u \in H_{0}^{\alpha / 2}(\Omega)$. Then, there exist positive constants $C_{1}<1$ and $C_{2}$ independent of $u$, such that

$$
C_{1}\|u\|_{\alpha / 2} \leq|u|_{\alpha / 2} \leq\|u\|_{\alpha / 2} \leq C_{2}|u|_{H^{\alpha / 2}(\Omega)} .
$$

The following lemma and remark summarize the properties of the interpolation operator $I_{N}$.

Lemma 8 (see [44]). Let $s \geq 1$. If $u \in H^{s}(\Omega)$, then there exists a constant $C>0$ independent of $N$, such that $\left\|u-I_{N} u\right\|_{l} \leq$ $C N^{l-s}\|u\|_{s}$, for any $0 \leq l \leq 1$.

Remark 9. A smooth solution of a fractional differential equation does not mean a smooth source term and vice versa. Therefore, the regularity order s of the solution $u$ is not the same as the regularity order $r$ of the source term $g$, i.e.,

$$
\left\|I_{N} g-g\right\| \leq C N^{-r}\|u\|_{r}, \quad \forall g \in H^{r}(\Omega) .
$$

Lemma 10 (see [45]). For any function $u(t)$ which is absolutely continuous on $[0, T]$, the following inequality is satisfied:

$$
\left(\frac{\partial^{\beta}}{\partial t^{\beta}} u(t), u(t)\right) \geq \frac{1}{2} \frac{\partial^{\beta}}{\partial t^{\beta}}\|u(t)\|^{2}
$$

Lemma 11 (see [46]). The discrete counterpart to the inequality (32) is given as

$$
\left(D_{\tau}^{\beta} u^{k}, u^{k}\right) \geq \frac{1}{2} D_{\tau}^{\beta}\left\|u^{k}\right\|^{2}
$$

such that $D_{\tau}^{\beta} u^{k}$ is the discrete time-fractional difference operator of the L1 type as defined in (13).

Plenty of researchers in recent years are stuck on the study of the continuous fractional Grönwall-type inequalities and their developments. However, the discrete fractional Grönwall-type inequality was far from well investigated, and more recently, the efforts paid in [47-50] tried to fill that gap. In what follows, we present recent discrete fractionaltype inequalities. These inequalities play an important role in analyzing stability and convergence of the L1-schemes for the multiterm problems with nonlinear delay.

Lemma 12 (discrete fractional Grönwall inequality [29]). Let $\left\{\phi^{i}\right\}_{i=-N_{\tilde{s}}}^{\infty}$ and $\left\{\xi_{l}\right\}_{l=0}^{\infty}$ be nonnegative sequences. Let $\varepsilon_{l}, \mu_{i}, \forall l$ $=0, \cdots, m, i \in \mathbb{Z}_{[1,6]}$ and $c_{0}$ be positive constants independent of $\tau$. The fractional orders are defined as $0<\beta_{0} \leq \beta_{1} \leq \cdots \leq$ $\beta_{m-1} \leq \beta_{m} \leq 1$. If $\phi^{i} \geq 0 \forall i \geq 0, \phi^{0}$ is known and $\phi^{i}=0$ if $i<0$,

$$
\begin{gathered}
\sum_{l=0}^{m} \varepsilon_{l} D_{\tau}^{\beta_{l}} \phi^{j} \leq \mu_{1} \phi^{j}+\varepsilon_{0}\left\|\xi^{j}\right\|^{2} \forall j \leq N_{\tilde{s}}, \\
\sum_{l=0}^{m} \varepsilon_{l} D_{\tau}^{\beta_{l}} \phi^{j} \leq \mu_{1} \phi^{j}+\mu_{2} \phi^{j-1}+\mu_{3} \phi^{j-2}+\mu_{4} \phi^{j-3}+\mu_{5} \phi^{j-N_{\tilde{s}}-1} \\
+\mu_{6} \phi^{j-N_{s}}+\varepsilon_{0}\left\|\xi^{j}\right\|^{2}, \forall j>N_{\tilde{s}},
\end{gathered}
$$

where $c_{0}$ and $\mu_{i}(i=1, \cdots, 6)$ are positive constants. Then, there exists a positive constant $\tau^{*} \geq \tau$ such that

$$
\phi^{n} \leq 2\left[\frac{c_{0} t_{n}^{\beta_{m}}}{\varepsilon_{m} \Gamma\left(1+\beta_{m}\right)}\left(\sum_{j=1}^{n}\left\|\xi^{j}\right\|^{2} \tau+\phi^{0} W\right)\right] E_{\beta_{m}}\left(\frac{2 \mu t_{n}^{\beta_{m}}}{\varepsilon_{m}}\right),
$$

where $E_{\beta}(z)=\sum_{k=0}^{\infty} z^{k} / \Gamma(1+k \beta)$ is the Mittag-Leffler function and

$$
\begin{aligned}
& \sum_{l=0}^{m} \varepsilon_{l} \frac{\Delta^{1-\beta_{l}}}{\Gamma\left(2-\beta_{l}\right)} \sum_{j=1}^{k} a_{j-1}^{\beta_{l}}:=W>0, \\
& \mu=\mu_{1}+\frac{\mu_{2}}{a_{0}^{\beta_{m}}-a_{1}^{\beta_{m}}}+\frac{\mu_{3}}{a_{1}^{\beta_{m}}-a_{2}^{\beta_{m}}}+\frac{\mu_{4}}{a_{2}^{\beta_{m}}-a_{3}^{\beta_{m}}} \\
& \quad+\frac{\mu_{5}}{a_{N_{\tilde{s}}-2}^{\beta_{m}}-a_{N_{\tilde{s}}}^{\beta_{m}}}+\frac{\mu_{6}}{a_{N_{\tilde{s}}-1}^{\beta_{m}}-a_{N_{\tilde{s}}}^{\beta_{m}}} .
\end{aligned}
$$




\section{Theoretical Analysis}

The purpose of this section is to study the efficiency of the fully discrete Galerkin spectral methods for (1) and (2). We start by stability analysis and gives theorem of stability in the first subsection. The second subsection is devoted to the convergence analysis, and the theorem of convergence is given there. For the theoretical analysis requirements, we assume that the function $f$ satisfies the following Lipschitz condition

$$
\left|f\left(u_{1}, v_{1}\right)-f\left(u_{2}, v_{2}\right)\right| \leq L\left(\left|u_{1}-u_{2}\right|+\left|v_{1}-v_{2}\right|\right)
$$

where $L$ is a positive constant.

5.1. Stability Analysis. The weak formulation of the scheme is as follows: find $\left\{u_{N}^{k}\right\}_{k=1}^{M} \in \mathscr{P}_{N}$, such that

$$
\begin{gathered}
\left(\sum_{r=0}^{m} q_{r} D_{\tau}^{\beta_{r}} u_{N}^{k}, v_{N}\right)+A\left(u_{N}^{k}, v_{N}\right) \\
=\left(I_{N} f\left(2 u_{N}^{k-1}-u_{N}^{k-2}, u_{N}^{k-N_{\tilde{s}}}\right), v_{N}\right) \\
\quad+\left(I_{N} g^{k}, v_{N}\right), \quad \forall v_{N} \in \mathscr{P}_{N},
\end{gathered}
$$

with

$$
u_{N}^{k}=\pi_{N}^{1,0} \varphi^{k}, \quad-N_{\tilde{s}} \leq k \leq 0 .
$$

It is a linear iterative scheme which means that we need only to get a solution to a system of linear equations at each time level. The well-posedness of that scheme is satisfied by the well-known Lax-Milgram lemma. Assume that $\left\{\tilde{u}_{N}^{k}\right\}_{k=1}^{M}$ is the solution of

$$
\begin{gathered}
\left(\sum_{r=0}^{m} q_{\mathrm{r}} D_{\tau}^{\beta_{r}} \tilde{u}_{N}^{k}, v_{N}\right)+A\left(\tilde{u}_{N}^{k}, v_{N}\right) \\
=\left(I_{N} f\left(2 \tilde{u}_{N}^{k-1}-\tilde{u}_{N}^{k-2}, \tilde{u}_{N}^{k-N_{\tilde{s}}}\right), v_{N}\right) \\
\quad+\left(I_{N} \tilde{g}^{k}, v_{N}\right), \quad \forall v_{N} \in \mathscr{P}_{N},
\end{gathered}
$$

with initial conditions

$$
\tilde{u}_{N}^{k}=\pi_{N}^{1,0} \varphi^{k}, \quad-N_{\tilde{s}} \leq k \leq 0 .
$$

Now, we present the theorem of stability in the following context.

Theorem 13. The fully discrete scheme (38) is unconditionally stable in the sense that for all $\tau>0$, the following holds:

$$
\left\|u_{N}^{k}-\tilde{u}_{N}^{k}\right\|^{2} \leq C \max _{1 \leq k \leq M}\left\|g^{k}-\tilde{g}^{k}\right\|^{2}
$$

Proof. Denote $\eta_{N}^{k}=u_{N}^{k}-\tilde{u}_{N}^{k}$. Subtracting (40) from (38), the following holds:

$$
\begin{aligned}
\left(\sum_{r=0}^{m} q_{r} D_{\tau}^{\beta_{r}} \eta_{N}^{k}, v_{N}\right)+A\left(\eta_{N}^{k}, v_{N}\right) \\
=\left(I_{N} f\left(2 u_{N}^{k-1}-u_{N}^{k-2}, u_{N}^{k-N_{\tilde{s}}}\right)\right. \\
\left.\quad-I_{N} f\left(2 \tilde{u}_{N}^{k-1}-\tilde{u}_{N}^{k-2}, \tilde{u}_{N}^{k-N_{\tilde{s}}}\right), v_{N}\right) \\
\quad+\left(I_{N} g^{k}-I_{N} \tilde{g}^{k}, v_{N}\right) .
\end{aligned}
$$

According to (37) and using the Hölder inequality and Young's inequality, we derive that

$$
\begin{aligned}
&\left(I_{N} f\left(2 u_{N}^{k-1}-u_{N}^{k-2}, u_{N}^{k-N_{\tilde{s}}}\right)-I_{N} f\left(2 \tilde{u}_{N}^{k-1}-\tilde{u}_{N}^{k-2}, \tilde{u}_{N}^{k-N_{\tilde{s}}}\right), v_{N}\right) \\
& \leq C L\left(\left\|2 \eta_{N}^{k-1}-\eta_{N}^{k-2}\right\|+\left\|\eta_{N}^{k-N_{\tilde{s}}}\right\|\right)\left\|v_{N}\right\| \\
& \leq \frac{\varepsilon}{2} C L^{2}\left\|2 \eta_{N}^{k-1}-\eta_{N}^{k-2}\right\|^{2}+\frac{\varepsilon}{2} C L^{2}\left\|\eta_{N}^{k-N_{\tilde{s}}}\right\|^{2}+\frac{1}{2 \varepsilon}\left\|v_{N}\right\|^{2} \\
& \leq 4 \varepsilon C L^{2}\left\|\eta_{N}^{k-1}\right\|^{2}+\varepsilon L^{2}\left\|\eta_{N}^{k-2}\right\|^{2}+\frac{\varepsilon}{2} C L^{2}\left\|\eta_{N}^{k-N_{\tilde{s}}}\right\|^{2} \\
&+\frac{1}{2 \varepsilon}\left\|v_{N}\right\|^{2}, \\
&\left(I_{N} g^{k}-I_{N} \tilde{g}^{k}, v_{N}\right) \leq \frac{\varepsilon}{2} C\left\|g^{k}-\tilde{g}^{k}\right\|^{2}+\frac{1}{2 \varepsilon}\left\|v_{N}\right\|^{2} .
\end{aligned}
$$

Then, (43) becomes

$$
\begin{aligned}
\left(\sum_{r=0}^{m} q_{r} D_{\tau}^{\beta_{r}} \eta_{N}^{k}, v_{N}\right)+A\left(\eta_{N}^{k}, v_{N}\right) & \\
\leq & \frac{1}{\varepsilon}\left\|v_{N}\right\|^{2}+4 \varepsilon C L^{2}\left\|\eta_{N}^{k-1}\right\|^{2}+\varepsilon L^{2}\left\|\eta_{N}^{k-2}\right\|^{2} \\
& +\frac{\varepsilon}{2} C L^{2}\left\|\eta_{N}^{k-N_{\tilde{s}}}\right\|^{2}+\frac{\varepsilon C}{2}\left\|g^{k}-\tilde{g}^{k}\right\|^{2} .
\end{aligned}
$$

Taking $v_{N}=\eta_{N}^{k}$ and using Lemma 11 and (27), we can deduce that

$$
\begin{aligned}
\sum_{r=0}^{m} \frac{q_{r}}{2} D_{\tau}^{\beta_{r}}\left\|\eta_{N}^{k}\right\|^{2}+|\eta|_{\alpha / 2}^{2} & \leq \frac{1}{\varepsilon}\left\|\eta_{N}^{k}\right\|^{2}+4 \varepsilon C L^{2}\left\|\eta_{N}^{k-1}\right\|^{2}+\varepsilon L^{2}\left\|\eta_{N}^{k-2}\right\|^{2} \\
& +\frac{\varepsilon}{2} C L^{2}\left\|\eta_{N}^{k-N_{\tilde{s}}}\right\|^{2}+\frac{\varepsilon C}{2}\left\|g^{k}-\tilde{g}^{k}\right\|^{2},
\end{aligned}
$$

namely,

$$
\begin{aligned}
\sum_{r=0}^{m} q_{r} D_{\tau}^{\beta_{r}}\left\|\eta_{N}^{k}\right\|^{2} \leq & \frac{2}{\varepsilon}\left\|\eta_{N}^{k}\right\|^{2}+8 \varepsilon C L^{2}\left\|\eta_{N}^{k-1}\right\|^{2}+2 \varepsilon L^{2}\left\|\eta_{N}^{k-2}\right\|^{2} \\
& +\varepsilon C L^{2}\left\|\eta_{N}^{k-N_{\tilde{s}}}\right\|^{2}+\varepsilon C\left\|g^{k}-\tilde{g}^{k}\right\|^{2}
\end{aligned}
$$


By means of Lemma 12 and since $\varepsilon>0$, there exists a positive constant $\tau^{*}=\sqrt[\beta m]{q_{m} /\left(2 \Gamma\left(2-\beta_{m}\right) 2 / \varepsilon\right)}$; when $\tau<\tau^{*}$, we have

$$
\left\|\eta_{N}^{k}\right\|^{2} \leq \frac{2 \varepsilon C t_{k}^{\beta_{m}}}{q_{m} \Gamma\left(1+\beta_{m}\right)} E_{\beta_{m}}\left(2 \mu t_{k}^{\beta_{m}} / q_{m}\right) \sum_{k=1}^{M}\left\|g^{k}-\tilde{g}^{k}\right\|^{2}
$$

with $\mu=2 / \varepsilon+\left(8 C \varepsilon L^{2} /\left(a_{0}^{\beta_{m}}-a_{1}^{\beta_{m}}\right)\right)+\left(2 C \varepsilon L^{2} /\left(a_{1}^{\beta_{m}}-a_{2}^{\beta_{m}}\right)\right)+$ $\left(C \varepsilon L^{2} /\left(a_{N_{s}-1}^{\beta_{m}}-a_{N_{s}}^{\beta_{m}}\right)\right)$. Thus, the scheme is unconditionally stable. $\square$

5.2. Convergence Analysis. In this section, we investigate the convergence of the fully discrete scheme (38) using error estimation.

Theorem 14. Let $\left\{u^{k}\right\}_{k=-N_{s}}^{M}$ be the exact solution of equation (1) and $\left\{u_{N}^{k}\right\}_{k=-N_{s}}^{M}$ be the solution of (38). Suppose that ${ }_{0}^{C} D_{t}^{\beta_{r}} u \in L^{\infty}\left(0, T ; H_{0}^{\alpha / 2}(\Omega) \cap H^{s}(\Omega)\right), u \in L^{\infty}\left(-s, T ; H^{s}(\Omega)\right)$; we have

$$
\left|u^{k}-u_{N}^{k}\right|_{\alpha / 2} \leq C\left(N^{(\alpha / 2)-s}+N^{-r}+\tau^{2-\beta_{m}}\right), \quad 1 \leq k \leq M,
$$

where $C$ is independent of $N$ and $\tau$.

Proof. Denote $u^{k}-u_{N}^{k}=e_{N}^{k}=\left(u^{k}-\pi_{N}^{\alpha / 2,0} u^{k}\right)+\left(\pi_{N}^{\alpha / 2,0} u^{k}-\right.$ $\left.u_{N}^{k}\right)=\Delta \tilde{e}_{N}^{k}+\hat{e}_{N}^{k}$. The weak formulation of equation (1) is

$$
\begin{aligned}
& \left(\sum_{r=0}^{m} q_{r 0}^{C} D_{t}^{\beta_{r}} u^{k}, v_{N}\right)+A\left(u^{k}, v_{N}\right) \\
& =\left(f\left(u^{k}, u^{k-N_{\tilde{s}}}\right), v_{N}\right)+\left(g^{k}, v_{N}\right) .
\end{aligned}
$$

Subtracting (38) from (50) and owing to the definition of orthogonal projection, the error equation satisfies

$$
\left(\sum_{r=0}^{m} q_{r} D_{\tau}^{\beta_{r}} \widehat{e}_{N}^{k}, v_{N}\right)+A\left(\widehat{e}_{N}, v_{N}\right) \triangleq R_{1}^{k}+R_{2}^{k}+R_{3}^{k}+R_{4}^{k}
$$

where

$$
\begin{gathered}
R_{1}^{k}=\left(I_{N} f\left(u^{k}, u^{k-N_{\tilde{s}}}\right)-I_{N} f\left(2 u_{N}^{k-1}-u_{N}^{k-2}, u_{N}^{k-N_{\tilde{s}}}\right), v_{N}\right), \\
R_{2}^{k}=\left(f\left(u^{k}, u^{k-N_{\tilde{s}}}\right)-I_{N} f\left(u^{k}, u^{k-N_{\tilde{s}}}\right), v_{N}\right), \\
R_{3}^{k}=\left(\sum_{r=0}^{m} q_{r}\left(D_{\tau}^{\beta_{r}} \pi_{N}^{\alpha / 2,0} u^{k}-{ }_{0}^{C} D_{t}^{\beta_{r}} u^{k}\right), v_{N}\right), \\
R_{4}^{k}=\left(g^{k}-I_{N} g^{k}, v_{N}\right) .
\end{gathered}
$$

We next estimate the right-hand terms $R_{1}^{k}, R_{2}^{k}, R_{3}^{k}$, and $R_{4}^{k}$. For the first term $R_{1}^{k}$,

$$
\begin{aligned}
R_{1}^{k}= & \left(I_{N} f\left(u^{k}, u^{k-N_{\tilde{s}}}\right)-I_{N} f\left(2 u^{k-1}-u^{k-2}, u^{k-N_{\tilde{s}}}\right), v_{N}\right) \\
& +\left(I_{N} f\left(2 u^{k-1}-u^{k-2}, u^{k-N_{\tilde{s}}}\right)\right. \\
& \left.-I_{N} f\left(2 u_{N}^{k-1}-u_{N}^{k-2}, u_{N}^{k-N_{\tilde{s}}}\right), v_{N}\right) \triangleq R_{11}^{k}+R_{12}^{k} .
\end{aligned}
$$

By applying the Taylor expansion, the following holds:

$$
\begin{aligned}
f\left(u^{k}, u^{k-N_{\tilde{s}}}\right)= & f\left(2 u^{k-1}-u^{k-2}, u^{k-N_{s}}\right) \\
& +\left(u^{k}-2 u^{k-1}+u^{k-2}\right) f^{\prime}{ }_{1}\left(\xi, u^{k-N_{\tilde{s}}}\right. \\
= & f\left(2 u^{k-1}-u^{k-2}, u^{k-N_{\tilde{s}}}\right)+\tilde{c}_{u} \tau^{2} .
\end{aligned}
$$

Furthermore, by means of the Hölder inequality and Young's inequality, we have

$$
\begin{aligned}
R_{11}^{k} & \leq\left\|I_{N} f\left(u^{k}, u^{k-N_{\tilde{s}}}\right)-I_{N} f\left(2 u^{k-1}-u^{k-2}, u^{k-N_{\check{s}}}\right)\right\|\left\|v_{N}\right\| \\
& \leq C\left\|f\left(u^{k}, u^{k-N_{\tilde{s}}}\right)-f\left(2 u^{k-1}-u^{k-2}, u^{k-N_{\check{s}}}\right)\right\|\left\|v_{N}\right\| \\
& \leq \frac{\varepsilon}{2} \tilde{c}_{u} \tau^{4}+\frac{1}{2 \varepsilon}\left\|v_{N}\right\|^{2} .
\end{aligned}
$$

According to (37), we can deduce that

$$
\begin{aligned}
R_{12}^{k} \leq & L C\left(\left\|2 e_{N}^{k-1}-e_{N}^{k-2}\right\|+\left\|e_{N}^{k-N_{\tilde{s}}}\right\|\right)\left\|v_{N}\right\| \\
\leq & L C\left(\left\|2 \tilde{e}_{N}^{k-1}-\hat{e}_{N}^{k-2}\right\|+\left\|\hat{e}_{N}^{k-N_{\tilde{s}}}\right\|\right. \\
& \left.+\left\|2 \tilde{e}_{N}^{k-1}-\tilde{e}_{N}^{k-2}\right\|+\left\|\tilde{e}_{N}^{k-N_{\tilde{s}}}\right\|\right)\left\|v_{N}\right\| \\
\leq & \frac{8 \varepsilon}{2} C L^{2}\left\|\hat{e}_{N}^{k-1}\right\|^{2}+\frac{2 \varepsilon}{2} C L^{2}\left\|\hat{e}_{N}^{k-2}\right\|^{2} \\
& +\frac{\varepsilon}{2} L^{2}\left\|\hat{e}_{N}^{k-N_{\tilde{s}}}\right\|^{2}+\frac{8 \varepsilon}{2} C L^{2}\left\|\tilde{e}_{N}^{k-1}\right\|^{2} \\
& +\frac{2 \varepsilon}{2} C L^{2}\left\|\tilde{e}_{N}^{k-2}\right\|^{2}+\frac{\varepsilon}{2} C L^{2}\left\|\tilde{e}_{N}^{k-N_{s}}\right\|^{2}+\frac{1}{2 \varepsilon}\left\|v_{N}\right\|^{2} .
\end{aligned}
$$

Moreover, owing to Lemmas 6 and 7, the following holds:

$$
\begin{aligned}
\left\|\tilde{e}_{N}^{k-1}\right\|^{2} & \leq \frac{C}{C_{1}} N^{\alpha-2 s}\left\|u^{k-1}\right\|_{s}^{2},\left\|\tilde{e}_{N}^{k-2}\right\|^{2} \\
& \leq \frac{C}{C_{1}} N^{\alpha-2 s}\left\|u^{k-2}\right\|_{s}^{2},\left\|\tilde{e}_{N}^{k-N_{\tilde{s}}}\right\|^{2} \\
& \leq \frac{C}{C_{1}} N^{\alpha-2 s}\left\|u^{k-N_{s}}\right\|_{s}^{2} .
\end{aligned}
$$


Then, (56) becomes

$$
\begin{aligned}
R_{12}^{k} \leq & 4 \varepsilon C L^{2}\left\|\hat{e}_{N}^{k-1}\right\|^{2}+\varepsilon C L^{2}\left\|\widehat{e}_{N}^{k-2}\right\|^{2}+\frac{\varepsilon}{2} C L^{2}\left\|\widehat{e}_{N}^{k-N_{\tilde{s}}}\right\|^{2} \\
& +\frac{C}{C_{1}} N^{\alpha-2 s}\|u\|_{L^{\infty}\left(-s, T ; H^{s}(\Omega)\right)}^{2}+\frac{1}{2 \varepsilon}\left\|v_{N}\right\|^{2} .
\end{aligned}
$$

Substituting (55) and (58) into (53), we can derive that

$$
\begin{aligned}
R_{1}^{k} \leq & \frac{1}{\varepsilon}\left\|v_{N}\right\|^{2}+4 \varepsilon C L^{2}\left\|\widehat{e}_{N}^{k-1}\right\|^{2}+\varepsilon C L^{2}\left\|\widehat{e}_{N}^{k-2}\right\|^{2} \\
& +\frac{\varepsilon}{2} C L^{2}\left\|\widehat{e}_{N}^{k-N_{\tilde{s}}}\right\|^{2}+\frac{C}{C_{1}} N^{\alpha-2 s}\|u\|_{L^{\infty}\left(-s, T ; H^{s}(\Omega)\right)}^{2}+\frac{\varepsilon}{2} \tilde{c}_{u} \tau^{4} .
\end{aligned}
$$

For the second term $R_{2}^{k}$, by means of the Hölder inequality, the following holds:

$$
R_{2}^{k} \leq \frac{\varepsilon}{2} C N^{-2 r}\|u\|_{L^{\infty}\left(-s, T ; H^{s}(\Omega)\right)}^{2}+\frac{1}{2 \varepsilon}\left\|v_{N}\right\|^{2} .
$$

For the third term $R_{3}^{k}$, the following holds:

$$
\begin{aligned}
R_{3}^{k}= & \left(\sum_{r=0}^{m} q_{r}\left(D_{\tau}^{\beta_{r}} \pi_{N}^{\alpha / 2,0} u^{k}-{ }_{0}^{C} D_{t}^{\beta_{r}} \pi_{N}^{\alpha / 2,0} u^{k}\right), v_{N}\right) \\
& +\left(\sum_{r=0}^{m} q_{r}\left({ }_{0}^{C} D_{t}^{\beta_{r}} \pi_{N}^{\alpha / 2,0} u^{k}-{ }_{0}^{C} D_{t}^{\beta_{r}} u^{k}\right), v_{N}\right) \\
= & \left(\pi_{N}^{\alpha / 2,0} \sum_{r=0}^{m} q_{r}\left(D_{\tau}^{\beta_{r}} u^{k}-{ }_{0}^{C} D_{t}^{\beta_{r}} u^{k}\right), v_{N}\right) \\
& -\left(\sum_{r=0}^{m} q_{r 0}^{C} D_{t}^{\beta_{r}} \tilde{e}_{N}^{k}, v_{N}\right) \triangleq R_{31}^{k}+R_{32}^{k} .
\end{aligned}
$$

Using (12) and the Hölder inequality, the following holds:

$$
\begin{aligned}
R_{31}^{k} & \leq \frac{\varepsilon}{2} \sum_{r=0}^{m} q_{r}\left\|\pi_{N}^{\alpha / 2,0}\left(D_{\tau}^{\beta_{r}} u^{k}-{ }_{0}^{C} D_{t}^{\beta_{r}} u^{k}\right)\right\|^{2}+\frac{1}{2 \varepsilon}\left\|v_{N}\right\|^{2} \\
& \leq \frac{\varepsilon}{2} C \sum_{r=0}^{m} q_{r}\left\|\left(D_{\tau}^{\beta_{r}} u^{k}-{ }_{0}^{C} D_{t}^{\beta_{r}} u^{k}\right)\right\|^{2}+\frac{1}{2 \varepsilon}\left\|v_{N}\right\|^{2} \\
& \leq \frac{\varepsilon}{2} C_{1, u} \tau^{4-2 \beta_{m}}+\frac{1}{2 \varepsilon}\left\|v_{N}\right\|^{2} .
\end{aligned}
$$

Furthermore, according to Lemma 2, we have

$$
\begin{aligned}
R_{32}^{k} & \leq \frac{\varepsilon}{2} C N^{\alpha-2 s} \sum_{r=0}^{m} q_{r}\left\|{ }_{0}^{C} D_{t}^{\beta_{r}} u^{k}\right\|_{s}^{2}+\frac{1}{2 \varepsilon}\left\|v_{\mathrm{N}}\right\|^{2} \\
& \leq \frac{\varepsilon}{2} C N^{\alpha-2 s} \sum_{r=0}^{m} q_{r}\left\|{ }_{0}^{C} D_{t}^{\beta_{r}} u\right\|_{L^{\infty}\left(-s, T ; H^{s}(\Omega)\right)}^{2}+\frac{1}{2 \varepsilon}\left\|v_{N}\right\|^{2} .
\end{aligned}
$$

Thus, (61) becomes

$$
\begin{aligned}
R_{3}^{k} \leq & \frac{\varepsilon}{2} C N^{\alpha-2 s} \sum_{r=0}^{m} q_{r}\left\|{ }_{0}^{C} D_{t}^{\beta_{r}} u\right\|_{L^{\infty}\left(-s, T ; H^{s}(\Omega)\right)}^{2} \\
& +\frac{\varepsilon}{2} C_{2, u} \tau^{4-2 \beta_{m}}+\frac{1}{\varepsilon}\left\|v_{N}\right\|^{2}
\end{aligned}
$$

For the fourth term $R_{4}^{k}$, the following holds by invoking Remark 9:

$$
R_{4}^{k} \leq \frac{\varepsilon}{2} C N^{\alpha-2 r}\|u\|_{L^{\infty}\left(-s, T ; H^{r}(\Omega)\right)}^{2}+\frac{1}{2 \varepsilon}\left\|v_{N}\right\|^{2} .
$$

Substituting (59), (60), (64), and (65) into (51), we can infer that

$$
\begin{aligned}
\left(\sum_{r=0}^{m} q_{r} D_{\tau}^{\beta_{r}} \widehat{e}_{N}^{k}, v_{N}\right)+A\left(\widehat{e}_{N}, v_{N}\right) \\
\leq \leq \frac{5}{2 \varepsilon}\left\|v_{N}\right\|^{2}+4 \varepsilon C L^{2}\left\|\widehat{e}_{N}^{k-1}\right\|^{2}+\varepsilon C L^{2}\left\|\widehat{e}_{N}^{k-2}\right\|^{2} \\
\quad+\frac{\varepsilon}{2} C L^{2}\left\|\widehat{e}_{N}^{k-N_{\tilde{s}}}\right\|^{2}+\tilde{\mathscr{G}},
\end{aligned}
$$

where

$$
\begin{aligned}
\tilde{\mathscr{G}}= & \varepsilon \tilde{C} N^{\alpha-2 s}\left(\sum_{r=0}^{m} q_{r}\left\|{ }_{0}^{C} D_{t}^{\beta_{r}} u\right\|_{L^{\infty}\left(0, T ; H^{s}(\Omega)\right)}^{2}+\|u\|_{L^{\infty}\left(-s, T ; H^{s}(\Omega)\right)}^{2}\right) \\
& +\varepsilon \tilde{C} N^{-2 r}\|u\|_{L^{\infty}\left(-s, T ; H^{r}(\Omega)\right)}^{2}+\varepsilon \tilde{C}_{u} \tau^{4-2 \beta_{m}} .
\end{aligned}
$$

Taking $v_{N}=\widehat{e}_{N}^{k}$ in (66) and applying Lemma 11, we can conclude that

$$
\begin{aligned}
\sum_{r=0}^{m} \frac{q_{r}}{2} D_{\tau}^{\beta_{r}}\left\|\widehat{e}_{N}^{k}\right\|^{2}+\left|\hat{e}_{N}^{k}\right|_{\alpha / 2}^{2} \\
\leq \frac{5}{2 \varepsilon}\left\|\widehat{e}_{N}^{k}\right\|^{2}+4 \varepsilon C L^{2}\left\|\hat{e}_{N}^{k-1}\right\|^{2}+\varepsilon C L^{2}\left\|\hat{e}_{N}^{k-2}\right\|^{2} \\
\quad+\frac{\varepsilon}{2} C L^{2}\left\|\hat{e}_{N}^{k-N_{\tilde{s}}}\right\|^{2}+\tilde{\mathscr{G}},
\end{aligned}
$$

namely,

$$
\begin{aligned}
\sum_{r=0}^{m} \frac{q_{r}}{2} D_{\tau}^{\beta_{r}}\left\|\hat{e}_{N}^{k}\right\|^{2} \leq & \frac{5}{\varepsilon}\left\|\hat{e}_{N}^{k}\right\|^{2}+8 \varepsilon C L^{2}\left\|\widehat{e}_{N}^{k-1}\right\|^{2}+2 \varepsilon C L^{2}\left\|\hat{e}_{N}^{k-2}\right\|^{2} \\
& +\varepsilon C L^{2}\left\|\widehat{e}_{N}^{k-N_{\tilde{s}}}\right\|^{2}+\mathscr{G}
\end{aligned}
$$


TABle 1: The $L^{2}$-errors and their convergence orders versus $\tau$ and $\alpha$ at $N=50$ for Example 15 .

\begin{tabular}{|c|c|c|c|c|c|c|}
\hline \multirow{2}{*}{$\tau$} & \multicolumn{2}{|c|}{$\alpha=1.1$} & \multicolumn{2}{|c|}{$\alpha=1.5$} & \multicolumn{2}{|c|}{$\alpha=1.9$} \\
\hline & Error & Order & Error & Order & Error & Order \\
\hline $1.5 / 50$ & $5.057 \times 10^{-5}$ & - & $4.692 \times 10^{-5}$ & - & $4.163 \times 10^{-5}$ & - \\
\hline $1.5 / 100$ & $1.927 \times 10^{-5}$ & 1.392 & $1.989 \times 10^{-5}$ & 1.238 & $1.783 \times 10^{-5}$ & 1.223 \\
\hline $1.5 / 150$ & $1.094 \times 10^{-5}$ & 1.395 & $1.192 \times 10^{-5}$ & 1.262 & $1.086 \times 10^{-5}$ & 1.224 \\
\hline $1.5 / 200$ & $7.327 \times 10^{-6}$ & 1.396 & $8.283 \times 10^{-6}$ & 1.267 & $7.592 \times 10^{-6}$ & 1.244 \\
\hline $1.5 / 250$ & $5.365 \times 10^{-6}$ & 1.396 & $6.226 \times 10^{-6}$ & 1.279 & $5.732 \times 10^{-6}$ & 1.259 \\
\hline $1.5 / 300$ & $4.159 \times 10^{-6}$ & 1.397 & $4.933 \times 10^{-6}$ & 1.277 & $4.560 \times 10^{-6}$ & 1.255 \\
\hline $2-\beta_{5}$ & - & 1.333 & - & 1.333 & - & 1.333 \\
\hline
\end{tabular}

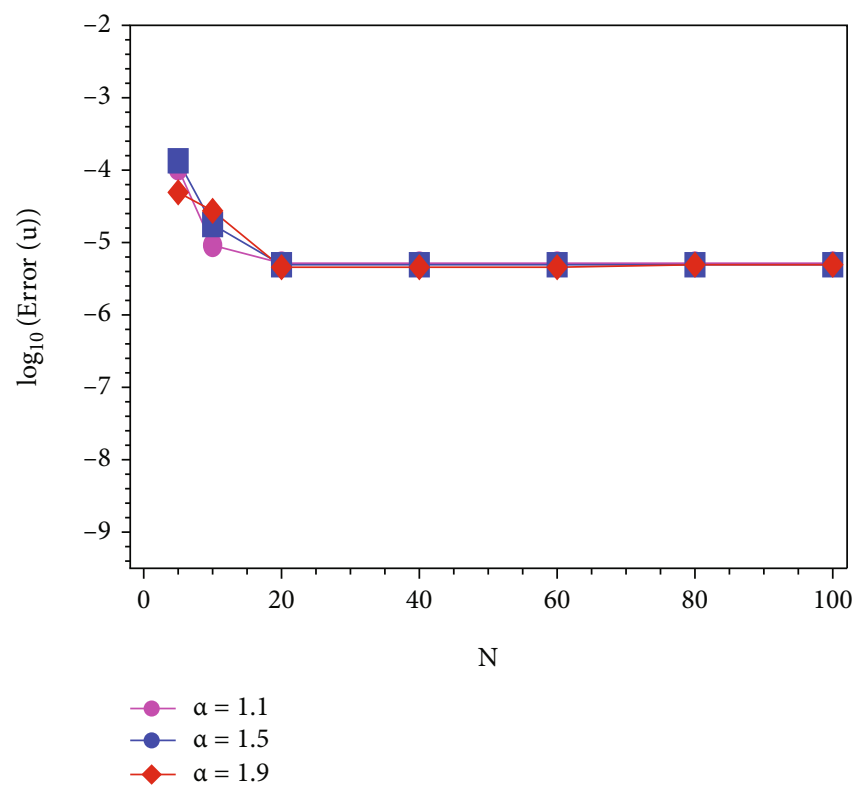

FIgURE 1: Convergence order in space direction for different values of $\alpha$ at $\tau=1.5 / 300$.

with $\mathscr{G}=2 \tilde{\mathscr{G}}$. By means of Lemma 12 and since $\varepsilon>0$, there exists a positive constant $\tau^{*}=\sqrt[\beta m]{q_{m} /\left(2 \Gamma\left(2-\beta_{m}\right) 10 / \varepsilon\right)}$; when $\tau<\tau^{*}$, we have

$$
\left\|\hat{e}_{N}^{k}\right\|^{2} \leq \frac{2 G t_{k}^{\beta_{m}}}{q_{m} \Gamma\left(1+\beta_{m}\right)} E_{\beta_{m}}\left(\frac{2 \mu t_{k}^{\beta_{m}}}{q_{m}}\right),
$$

with $\mu=10 / \varepsilon+\left(16 \varepsilon C L^{2} /\left(a_{0}^{\beta_{m}}-a_{1}^{\beta_{m}}\right)\right)+\left(4 C \varepsilon L^{2} /\left(a_{1}^{\beta_{m}}-a_{2}^{\beta_{m}}\right)\right)$ $+\left(2 C \varepsilon L^{2} /\left(a_{N_{s}-1}^{\beta_{m}}-a_{N_{s}}^{\beta_{m}}\right)\right)$. Thus, the scheme is unconditionally convergent. Finally, by means of the triangle inequality and Lemma 6, we complete the proof of (49).

\section{Numerical Experiments}

Some numerical experiments are performed here to clarify the convergence orders of the considered scheme in time and space. We also show the impact of time- and spacefractional orders on the behaviour of the dynamics for the solution of nonlinear delay reaction diffusion equations. To examine the temporal and spatial convergence orders sepa- rately, the orders of convergence in time and space shall be determined from the $L^{2}$-error norms defined as

$$
\text { Order }=\frac{\ln \left(\left\|e\left(N, M_{1}\right)\right\| /\left\|e\left(N, M_{2}\right)\right\|\right)}{\ln \left(M_{1} / M_{2}\right)},
$$

where $M_{1} \neq M_{2}$.

Example 15. Consider the nonlinear delay reaction-diffusion problem

$$
\begin{aligned}
\sum_{r=1}^{Q} \frac{\partial^{\beta_{r}} u}{\partial t^{\beta_{r}}}(x, t)= & \frac{\partial^{\alpha} u}{\partial|x|^{\alpha}}(x, t)-2 u(x, t)+\frac{u(x, t-1.5)}{1+u^{2}(x, t-1.5)} \\
& +g(x, t), x \in(0,1), t \in(0,1] .
\end{aligned}
$$

We choose the fractional orders $\beta_{r}=(2 Q+r-5) / 3 Q$. The source function $g(x, t)$ is given such that problem (72) has the exact solution $t^{\beta_{5}+1} x^{2}(1-x)^{2}$. 

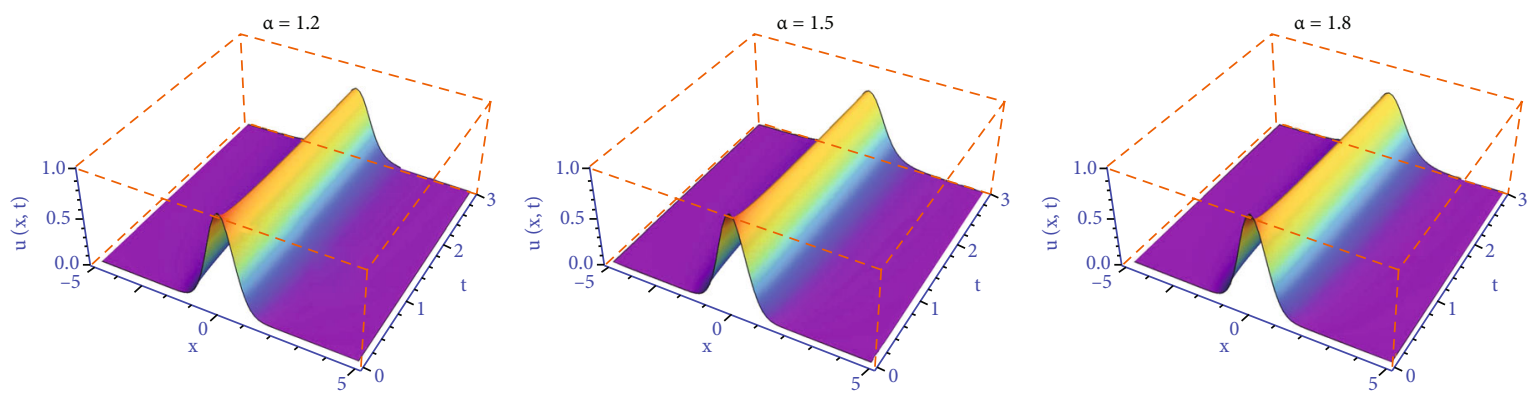

Figure 2: Evaluations of $u$ for different fractional-order parameters $\alpha$.
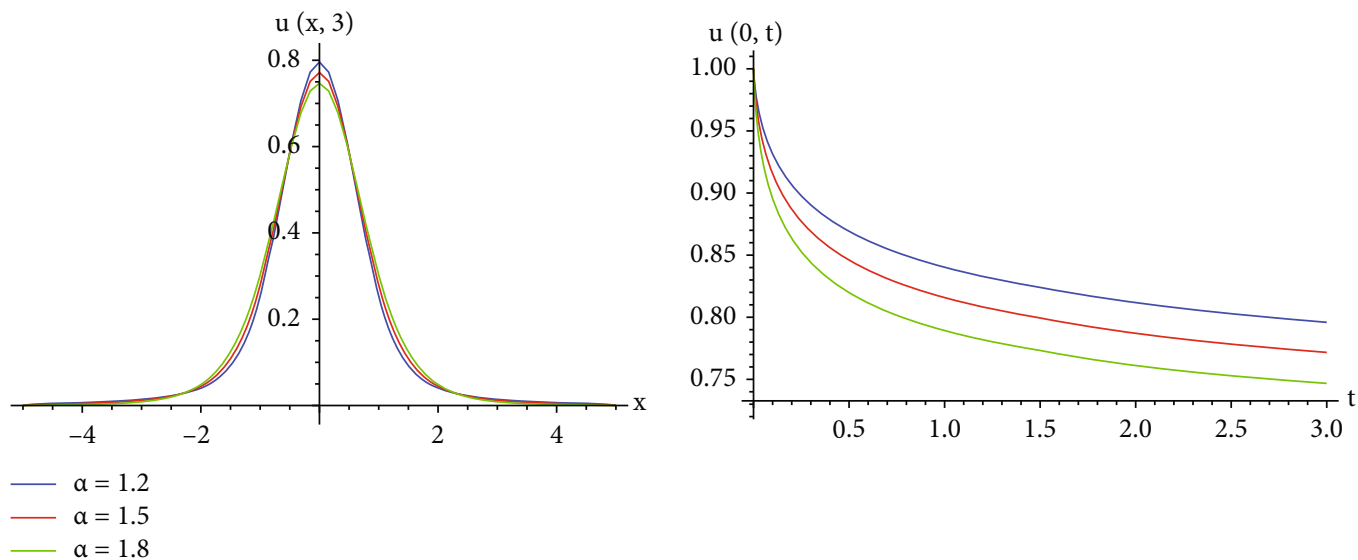

FIgURE 3: Evaluations of $u$ at $x=0$ and $t=T$ for different fractional-order parameters $\alpha$.

In Table 1 , we list the $L^{2}$-errors and corresponding convergence orders with $\alpha=1.1,1.5,1.9$ and $N=50$ for $Q=5$. We can see that these results confirm the expected theoreti$\mathrm{cal}$ order convergence in time. The convergence orders in space are depicted for different values of $\alpha$ and $\beta$ at $M=$ 500 in Figure 1. All the convergence results are in agreement with the theoretical results.

Example 16. We consider the following fractional problem, where the dynamics of the solution is very interesting and the exact solution is unknown:

$$
\begin{aligned}
\sum_{r=1}^{8} \frac{\partial^{\beta_{r}} u}{\partial t^{\beta_{r}}}(x, t)= & \frac{\partial^{\alpha} u}{\partial|x|^{\alpha}}(x, t)+u(x, t)(1-u(x, t)) \\
& \cdot(1+u(x, t-1.5)), \quad x \in(a, b), t \in(0,3]
\end{aligned}
$$

with the initial value $u(x, 0)=e^{-2 x^{2}}$ and $\beta_{r}=r / 10$.

Figures 2 and 3 show the profiles of the numerical solution with the fractional-order parameters $\alpha=1.2,1.5,1.8$ with $N=100$ and $\tau=1.5 / 500$. We can observe that the fractional-order parameter $\alpha$ affects the shape of the solutions. We can say that the fractional-order parameters can be used in physics to modify the shape of waves without changing the nonlinearity and dispersion effects of the fractional nonlinear problems.

\section{Conclusion and Remarks}

We have constructed and analyzed a novel explicit finite difference/Galerkin-Legendre spectral scheme for the nonlinear multiterm Riesz space and Caputo time fractional reaction-diffusion equation with delay. The problem was first approximated by the $L 1$ difference method on the temporal direction, and then, the Galerkin-Legendre spectral method was applied on the spatial discretization. Using an appropriate form of discrete fractional Grönwall inequality, the stability and the convergence of the fully discrete scheme were investigated. We have proven that the proposed method is stable and has a convergent order $2-\beta_{m}$ in time and an exponential rate of convergence in space. Highorder difference schemes can be handled to raise the temporal convergence order. This can be done by using the Alikhanov scheme [46], and it can be designed theoretically and numerically easily as shown in our context. Two numerical examples are given to show that the numerical results are consistent with theoretical ones in the case of the smoothness of the solution with respect to time and space.

\section{Data Availability}

The data used to support the findings of this study are included within the article.

\section{Conflicts of Interest}

The authors declare that they have no competing interests. 


\section{Authors' Contributions}

A. K. Omran contributed to the conceptualization, writing of the original draft, data curation, figure preparation, and methodology. M. A. Zaky and A.S. Hendy reviewed and edited the manuscript and carried out the experiments, formal analysis, and supervision. V. G. Pimenov contributed to the conceptualization, writing of the original draft, data curation, figure preparation, methodology, and supervision.

\section{Acknowledgments}

A. K. Omran is funded by a scholarship under the joint executive program between the Arab Republic of Egypt and Russian Federation. M. A. Zaky wishes to acknowledge the support of the Nazarbayev University Program (091019CRP2120). M. A. Zaky wishes also to acknowledge the partial support of the Science Committee of the Ministry of Education and Science of the Republic of Kazakhstan (Grant "Dynamical Analysis and Synchronization of Complex Neural Networks with Its Applications”).

\section{References}

[1] M. Abbaszadeh and M. Dehghan, "A class of moving kriging interpolation-based DQ methods to simulate multidimensional space Galilei invariant fractional advectiondiffusion equation," Numerical Algorithms, pp. 1-29, 2021.

[2] M. Abbaszadeh and M. Dehghan, "Fourth-order alternating direction implicit difference scheme to simulate the spacetime Riesz tempered fractional diffusion equation," International Journal of Computer Mathematics, vol. 98, no. 11, pp. 2137-2155, 2021.

[3] M. Abbaszadeh, H. Pourbashash, and M. Khaksar-e Oshagh, "The local meshless collocation method for solving 2D fractional Klein-Kramers dynamics equation on irregular domains," International Journal of Numerical Methods for Heat \& Fluid Flow, 2021.

[4] A. S. Hendy and M. A. Zaky, "Combined Galerkin spectral/ finite difference method over graded meshes for the generalized nonlinear fractional Schrödinger equation," Nonlinear Dynamics, vol. 103, no. 3, pp. 2493-2507, 2021.

[5] A. S. Hendy, M. A. Zaky, R. M. Hafez, and R. H. De Staelen, "The impact of memory effect on space fractional strong quantum couplers with tunable decay behavior and its numerical simulation," Scientific Reports, vol. 11, no. 1, article 10275, 2021.

[6] E. Loghman, A. Kamali, F. Bakhtiari-Nejad, M. Abbaszadeh, and M. Amabili, "On the combined shooting-pseudoarclength method for finding frequency response of nonlinear fractional-order differential equations," Journal of Sound and Vibration, vol. 516, article 116521, 2022.

[7] M. A. Zaky and A. S. Hendy, "Convergence analysis of anL1continuous Galerkin method for nonlinear time-space fractional Schrödinger equations," International Journal of Computer Mathematics, vol. 98, no. 7, pp. 1420-1437, 2021.

[8] M. A. Zaky, A. S. Hendy, and J. E. Macías-Díaz, "Semi-implicit Galerkin-Legendre spectral schemes for nonlinear time-space fractional diffusion-reaction equations with smooth and nonsmooth solutions," Journal of Scientific Computing, vol. 82, no. 1, pp. 1-27, 2020.
[9] M. Benchohra and F. Berhoun, "Impulsive fractional differential equations with state-dependent delay," Communications in Applied Analysis, vol. 14, no. 2, p. 213, 2010.

[10] C. Beta, M. Bertram, A. S. Mikhailov, H. H. Rotermund, and G. Ertl, "Controlling turbulence in a surface chemical reaction by time-delay autosynchronization," Physical Review E, vol. 67, no. 4, article 046224, 2003.

[11] V. Keyantuo, C. Lizama, and M. Warma, "Asymptotic behavior of fractional order semilinear evolution equations," Differential and Integral Equations, vol. 26, no. 7-8, pp. 757-780, 2013.

[12] V. T. Luong, "Decay mild solutions for two-term time fractional differential equations in Banach spaces," Journal of Fixed Point Theory and Applications, vol. 18, no. 2, pp. 417432, 2016.

[13] M. Dehghan and M. Abbaszadeh, "An efficient technique based on finite difference/finite element method for solution of two-dimensional space/multi-time fractional Bloch-Torrey equations," Applied Numerical Mathematics, vol. 131, pp. 190-206, 2018.

[14] Z. Liu, F. Liu, and F. Zeng, "An alternating direction implicit spectral method for solving two dimensional multi-term time fractional mixed diffusion and diffusion-wave equations," Applied Numerical Mathematics, vol. 136, pp. 139-151, 2019.

[15] K. Van Bockstal, "Existence and uniqueness of a weak solution to a non-autonomous time-fractional diffusion equation (of distributed order)," Applied Mathematics Letters, vol. 109, article 106540, 2020.

[16] J. F. Kelly, R. J. McGough, and M. M. Meerschaert, “Analytical time-domain Green's functions for power-law media," The Journal of the Acoustical Society of America, vol. 124, no. 5, pp. 2861-2872, 2008.

[17] J. Chen, F. Liu, V. Anh, S. Shen, Q. Liu, and C. Liao, “The analytical solution and numerical solution of the fractional diffusion-wave equation with damping," Applied Mathematics and Computation, vol. 219, no. 4, pp. 1737-1748, 2012.

[18] D. Vieru, C. Fetecau, and C. Fetecau, "Flow of a viscoelastic fluid with the fractional Maxwell model between two side walls perpendicular to a plate," Applied Mathematics and Computation, vol. 200, no. 1, pp. 459-464, 2008.

[19] L. Feng, F. Liu, I. Turner, and P. Zhuang, "Numerical methods and analysis for simulating the flow of a generalized OldroydB fluid between two infinite parallel rigid plates," International Journal of Heat and Mass Transfer, vol. 115, pp. 1309-1320, 2017.

[20] V. Daftardar-Gejji and S. Bhalekar, "Boundary value problems for multi-term fractional differential equations," Journal of Mathematical Analysis and Applications, vol. 345, no. 2, pp. 754-765, 2008.

[21] Y. Luchko, "Initial-boundary-value problems for the generalized multi-term time-fractional diffusion equation," Journal of Mathematical Analysis and Applications, vol. 374, no. 2, pp. 538-548, 2011.

[22] H. Jiang, F. Liu, I. Turner, and K. Burrage, “Analytical solutions for the multi-term time-fractional diffusion-wave/diffusion equations in a finite domain," Computers \& Mathematics with Applications, vol. 64, no. 10, pp. 3377-3388, 2012.

[23] X.-L. Ding and Y.-L. Jiang, "Analytical solutions for the multiterm time-space fractional advection-diffusion equations with mixed boundary conditions," Nonlinear Analysis: Real World Applications, vol. 14, no. 2, pp. 1026-1033, 2013. 
[24] Z. Li, Y. Liu, and M. Yamamoto, "Initial-boundary value problems for multi-term time-fractional diffusion equations with positive constant coefficients," Applied Mathematics and Computation, vol. 257, pp. 381-397, 2015.

[25] M. A. Zaky, "A Legendre spectral quadrature tau method for the multi-term time-fractional diffusion equations," Computational and Applied Mathematics, vol. 37, no. 3, pp. 3525-3538, 2018.

[26] A. S. Hendy, "Numerical treatment for after-effected multiterm time-space fractional advection-diffusion equations," Engineering with Computers, vol. 37, pp. 1-11, 2021.

[27] A. S. Hendy and R. H. De Staelen, "Theoretical analysis (convergence and stability) of a difference approximation for multiterm time fractional convection diffusion-wave equations with delay," Mathematics, vol. 8, no. 10, p. 1696, 2020.

[28] A. S. Hendy and M. A. Zaky, "Graded mesh discretization for coupled system of nonlinear multi-term time-space fractional diffusion equations," Engineering with Computers, pp. 1-13, 2020.

[29] M. A. Zaky, A. S. Hendy, A. A. Alikhanov, and V. G. Pimenov, "Numerical analysis of multi-term time-fractional nonlinear subdiffusion equations with time delay: what could possibly go wrong?", Communications in Nonlinear Science and Numerical Simulation, vol. 96, article 105672, 2021.

[30] A. S. Hendy, M. A. Zaky, and R. H. De Staelen, "A general framework for the numerical analysis of high-order finite difference solvers for nonlinear multi-term time-space fractional partial differential equations with time delay," Applied Numerical Mathematics, vol. 169, pp. 108-121, 2021.

[31] A. S. Hendy, M. A. Zaky, and D. Suragan, "Discrete fractional stochastic Gronwall inequalities arising in the numerical analysis of multi-term fractional order stochastic differential equations," Mathematics and Computers in Simulation, vol. 193, pp. 269-279, 2022.

[32] C. F. Lorenzo and T. T. Hartley, "Variable order and distributed order fractional operators," Nonlinear Dynamics, vol. 29, no. 1-4, pp. 57-98, 2002.

[33] M. Zheng, F. Liu, V. Anh, and I. Turner, "A high-order spectral method for the multi-term time-fractional diffusion equations," Applied Mathematical Modelling, vol. 40, no. 7-8, pp. 4970-4985, 2016.

[34] I. Podlubny, Fractional Differential Equations: An Introduction to Fractional Derivatives, Fractional Differential Equations, to Methods of Their Solution and Some of their Applications, vol. volume 198, Elsevier, 1998.

[35] D. Wang, A. Xiao, and W. Yang, "Crank-Nicolson difference scheme for the coupled nonlinear Schrodinger equations with the Riesz space fractional derivative," Journal of Computational Physics, vol. 242, pp. 670-681, 2013.

[36] V. J. Ervin and J. P. Roop, "Variational solution of fractional advection dispersion equations on bounded domains in $\mathbb{R}^{d}$," Numerical Methods for Partial Differential Equations, vol. 23, no. 2, pp. 256-281, 2007.

[37] H. Abo-Gabal, M. A. Zaky, A. S. Hendy, and E. H. Doha, "Computational aspects of fractional Romanovski-Bessel functions," Computational and Applied Mathematics, vol. 40, no. 4, pp. 1-16, 2021.

[38] I. G. Ameen, M. A. Zaky, and E. H. Doha, "Singularity preserving spectral collocation method for nonlinear systems of fractional differential equations with the right-sided Caputo fractional derivative," Journal of Computational and Applied Mathematics, vol. 392, article 113468, 2021.

[39] I. G. Ameen, N. A. Elkot, M. A. Zaky, A. S. Hendy, and E. H. Doha, "A pseudo-spectral scheme for systems of two-point boundary value problems with left and right sided fractional derivatives and related integral equations," Computer Modeling in Engineering \& Sciences, vol. 128, no. 1, pp. 21-41, 2021.

[40] M. A. Zaky, H. Abo-Gabal, R. M. Hafez, and E. H. Doha, "Computational and theoretical aspects of RomanovskiBessel polynomials and their applications in spectral approximations," Numerical Algorithms, pp. 1-35, 2021.

[41] Y. Lin and C. Xu, "Finite difference/spectral approximations for the time-fractional diffusion equation," Journal of Computational Physics, vol. 225, no. 2, pp. 1533-1552, 2007.

[42] J. Shen, "Efficient spectral-Galerkin method I. Direct solvers of second-and fourth-order equations using Legendre polynomials," SIAM Journal on Scientific Computing, vol. 15, no. 6, pp. 1489-1505, 1994.

[43] F. Zeng, F. Liu, C. Li, K. Burrage, I. Turner, and V. Anh, "A Crank-Nicolson ADI spectral method for a two-dimensional Riesz space fractional nonlinear reaction-diffusion equation," SIAM Journal on Numerical Analysis, vol. 52, no. 6, pp. 2599-2622, 2014.

[44] J. Shen, T. Tang, and L.-L. Wang, Spectral Methods: Algorithms, Analysis and Applications, vol. volume 41, Springer Science \& Business Media, 2011.

[45] A. A. Alikhanov, "A priori estimates for solutions of boundary value problems for fractional-order equations," Differential Equations, vol. 46, no. 5, pp. 660-666, 2010.

[46] A. A. Alikhanov, "A new difference scheme for the time fractional diffusion equation," Journal of Computational Physics, vol. 280, pp. 424-438, 2015.

[47] D. Li, H.-L. Liao, W. Sun, J. Wang, and J. Zhang, "Analysis of L1-Galerkin FEMs for time-fractional nonlinear parabolic problems," Communications in Computational Physics, vol. 24, no. 1, pp. 86-103, 2018.

[48] L. Li, B. Zhou, X. Chen, and Z. Wang, "Convergence and stability of compact finite difference method for nonlinear time fractional reaction-diffusion equations with delay," Applied Mathematics and Computation, vol. 337, pp. 144-152, 2018.

[49] H.-l. Liao, D. Li, and J. Zhang, "Sharp error estimate of the nonuniform L1 formula for linear reaction-subdiffusion equations," SIAM Journal on Numerical Analysis, vol. 56, no. 2, pp. 1112-1133, 2018.

[50] H.-1. Liao, W. McLean, and J. Zhang, “A discrete Grönwall inequality with applications to numerical schemes for subdiffusion problems," SIAM Journal on Numerical Analysis, vol. 57, no. 1, pp. 218-237, 2019. 